\title{
Analyse semi-probabiliste de la capacité portante des fondations superficielles
}

\section{BELABED}

\section{BENCHEIKH}

Laboratoire de Génie civil

et Hydraulique

Université de Guelma

BP 401

Guelma, Algérie DrBelabed@yahoo.de
Les fondations superficielles sont largement utilisées dans le domaine de la construction. Létude de leur stabilité en particulier vis-à-vis du poinçonnement constitue un aspect primordial dans le but de construire des fondations et des ouvrages plus fiables et économiques. Dans cet article, on propose une analyse semi-probabiliste de la stabilité des fondations superficielles vis-à-vis du poinçonnement à l'état ultime basée sur l'Eurocode 7. Cette nouvelle approche prend en considération le caractère aléatoire des paramètres d'influence (géométrie, sol, surcharge), notamment celui des paramètres de cisaillement du sol. Une comparaison de cette approche semi-probabiliste avec des approches conventionnelles nationales (approches déterministes) est effectuée pour différents cas de chargement et différents types de sol.

Mots-clés : fondations superficielles, capacité portante, stabilité Eurocode 7, probabilité de rupture, indice de fiabilité.

\section{Semi-probabilistic analysis of the bearing capacity of the shallow foundations}

The shallow foundations are largely used in the field of construction. The study of their stability in particular bearing capacity constitutes a fundamental aspect with an aim of building foundations and even works more reliable and economic. In this paper, we propose a semi-probabilistic analysis of the bearing capacity of the shaliow foundations at the ultimate state based on the new Eurocode 7. This new approach takes into account the randomness of the parameters of influence (geometry, ground, loads), in particular of the soil strenght parameters. A comparison between this semi-probabilistic approach and conventional national approaches (deterministic approaches) is carried out for several cases of loading and types of soil.

Key words : shallow foundations, bearing capacity, stability, Eurocode 7, probability of failure, reliability index. 


\section{Introduction}

Depuis longtemps, on sait dimensionner des fondations et des ouvrages stables en augmentant la section ou la surface des éléments constituant l'interface avec le sol, et ceci à travers l'utilisation de facteurs de sécurité globaux empiriques déterminés arbitrairement par l'expérience. De ce fait, on obtient souvent des constructions surdimensionnées.

La nécessité de construire des ouvrages plus fiables et économicues a amené les ingénieurs à développer un nouveau concept de sécurité basé sur la théorie de la probabilité qui devrait satisfaire ces exigences. On utilise à cette fin des facteurs partiels de sécurité dérivés de méthodes probabilistes pour couvrir la dispersion aléatoire des paramètres influant sur la stabilité de l'ouvrage au lieu d'un facteur global de sécurité (concept conventionnel). La sécurité du système est exprimée par la probabilité de rupture $\mathrm{P}_{\mathrm{f}}$ ou par l'indice de fiabilité $\beta$ défini par Hasofer/Lind (Späthe, 1987 ; Magnan et Baghery, 1982). Ce nouveau concept permet en théorie une comparaison objective et rationnelle des différents modes de rupture d'une structure et même des différentes structures à travers un indice de fiabilité homogène et invariable, ce qui n'est pas le cas pour le concept conventionnel (Bencheikh, 2005 ; Belabed, 1995, 1999a et b). La calibration des codes réglementaires semi-probabilistes vise à atteindre cette homogénéité, mais elle n'est que partielle (JCSS, 2001 ; Vrouwenvelder, 2002).

L'objectif de ce travail est l'étude dans un premier temps de la stabilité semi-probabiliste des fondations superficielles vis-à-vis du poinçonnement à l'état ultime d'après l'Eurocode 7 (2004). Une étude comparative avec le concept global conventionnel de sécurité (approche déterministe) a été ensuite effectuée pour valoriser l'analyse semi-probabiliste.

L'étude probabiliste de la stabilité de tout ouvrage en génie civil, en particulier géotechnique (fondations, murs de soutènement, etc.), doit se faire dans l'ordre suivant (Nottrodt, 1990).

\section{A - Modèles mécaniques}

Recherche des différents modèles mécaniques caractérisant les différentes possibilités de rupture d'une structure, basés sur des essais et sur des constatations tirées de l'analyse d'ouvrages réels existants.

Formulation des équations d'état limite correspondant à ces modèles.

\section{B-Études paramétriques}

Dimensionnement de l'élément ou de l'ouvrage.

Identification des paramètres aléatoires et de leurs propriétés statistiques.

Calcul de l'indice de fiabilité $\beta$ ou de la probabilité de rupture $\mathrm{P}_{\mathrm{f}}$.

\section{2}

\section{Principe de l'approche probabiliste}

\section{1}

\section{Définition}

Le coefficient (facteur) de sécurité $F_{S}$ d'un ouvrage est défini classiquement comme le rapport de la résistance à la rupture (capacitél $R$ sur la sollicitation $S$ appliquée $: F_{S}=\frac{R}{S}$.

Dans les calculs avec l'approche probabiliste, on renonce à la notion classique du facteur global de sécurité $F_{S}$ et on considère $R$ et $S$ comme deux variables aléatoires possédant chacune une moyenne et un écart-type $\left(m_{1,}, \sigma_{R}\right)$ et $\left(m_{S}, \sigma_{s}\right)$. On applique ainsi des facteurs partiels de sécurité sur les sollicitations et sur les résistances. La différence entre les deux variables aléaloires $Z=R-S$ est appelée marge de sécurité. La marge de sécurité est évidemment une variable aléatoire (Belabed, 1996).

Un état limite est atteint lorsque $Z=R-S=0$. Cette équation est appelée équation d'état limite ou de performance. Il y a rupture lorsque $S$ devient supérieur ou égal à $R$, c'est-à-dire $Z \leq 0$, et on peut définir la probabilité de rupture comme (Späthe, 1987. Belabed, 1996):

$$
\mathrm{P}_{\mathrm{f}}=\mathrm{P}_{\mathrm{r}}[(\mathrm{R}-\mathrm{S}) \leq 0]=\mathrm{P}_{\mathrm{f}}(\mathrm{R} \leq \mathrm{S})
$$

Si $Z>0$, ou $R>S$, la structure est dite stable.

Lorsque les variables $R$ et $S$ suivent des lois normales de Gauss, la marge de sécurité $\mathrm{Z}=\mathrm{R}-\mathrm{S}$ suit également une loi normale de densité (Fig. 1):

$$
f_{z}(z)=\frac{1}{\sigma_{z} \cdot \sqrt{2 \pi}} \exp \left[-\frac{1}{2}\left(\frac{z-m_{z}}{\sigma_{z}}\right)^{2}\right]
$$

dont la moyenne $m_{\mathrm{Z}}$ et l'écart-type $\sigma_{\mathrm{Z}}$ sont :

$$
\begin{gathered}
m_{z}=m_{R}-m_{S} \\
\sigma_{Z}=\sqrt{\sigma_{R}^{2}+\sigma_{S}^{2}}
\end{gathered}
$$

La forme retenue pour les distributions de $\mathrm{R}$ et $\mathrm{S}$ a une influence non négligeable sur la probabilité de rupture ou de ruine.

La rupture est associée à la portion de la densité de probabilité de la marge de sécurité correspondant à une valeur négative de cette marge, c'est -à-dire à $\mathrm{Z}<0$ représentée par des hachures sur la figure 1. La surface hachurée est la probabilité de rupture ou de ruine $P_{f}$ calculée au moyen de l'intégrale (Späthe, 1987):

$$
P_{f}=\int_{-\infty}^{0} f_{z}(z) \cdot d z
$$

Au lieu de la probabilité de rupture $\mathrm{P}_{\mathrm{f}}$, Cornell (cité par Späthe, 1987 et Favre, 2004) exprime la sécurité par un indice de fiabilité $\beta$, qui se calcule d'après la formule suivante (Fig. 1):

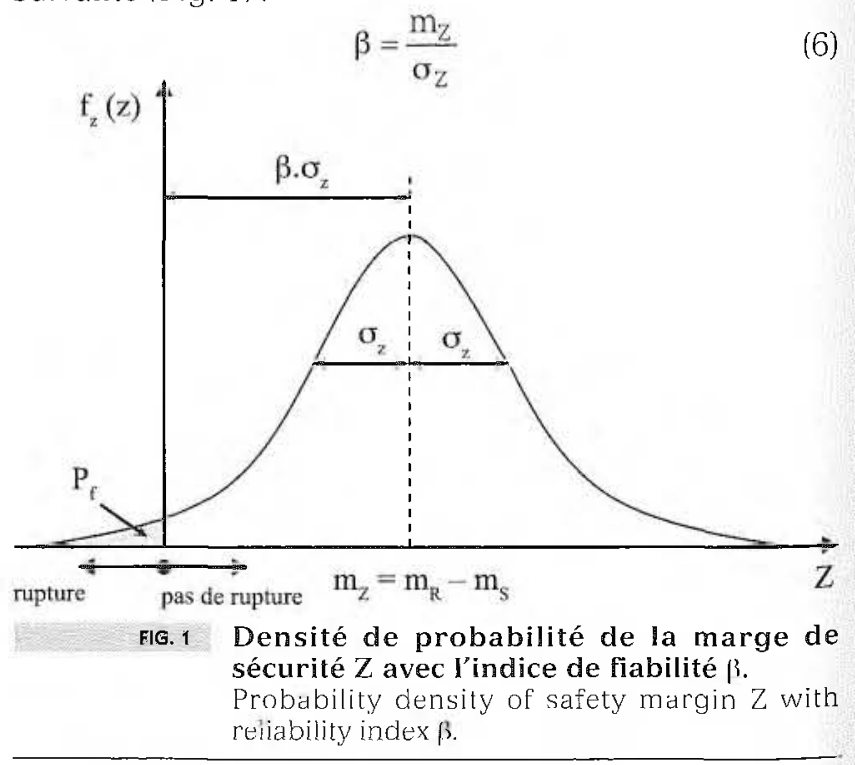


Il existe plusieurs méthodes mathématiques pour résoudre l'équation intégrale (5) : méthode de Rosenbluth, méthode des moments, théorie de la fiabilité de premier ordre (méthode FORM), théorie de la fiabilité de deuxième ordre (méthode SORM), méthode de Monte-Carlo, etc. Dans ce travail, l'analyse de la fiabilité a été effectuée à l'aide de la théorie de la fiabilité de premier ordre dont le principe est expliqué ci-dessous

\section{2}

\section{Théorie de la fiabilité de premier ordre (méthode FORM)}

L'exemple d'un cas simple de deux variables aléatoires $R$ et $S$ de distributions symétriques est illustré sur les figures 2 et 3 . Les densités de probabilités, $\mathrm{f}_{\mathrm{R}}(\mathrm{R})$ pour la capacité $R$ et $\mathrm{f}_{\mathrm{S}}(\mathrm{S})$ pour la sollicitation $\mathrm{S}$, sont représentées dans un système de coordonnées R-S (Fig. 2). Les courbes d'équiprobabilité, $f_{R}(R) \cdot f_{S}(S)=$ constante, ont une forme ellipsoidale. L'équation d'état limite $\mathrm{R}-\mathrm{S}=0$ est présentée dans le même système de coordonnées (Fig. 2). Elle divise le plan en une zone de sécurité $(R>S)$ et une zone de rupture $(R \leq S)$ (pour plus de détails, voir Späthe, 1987).

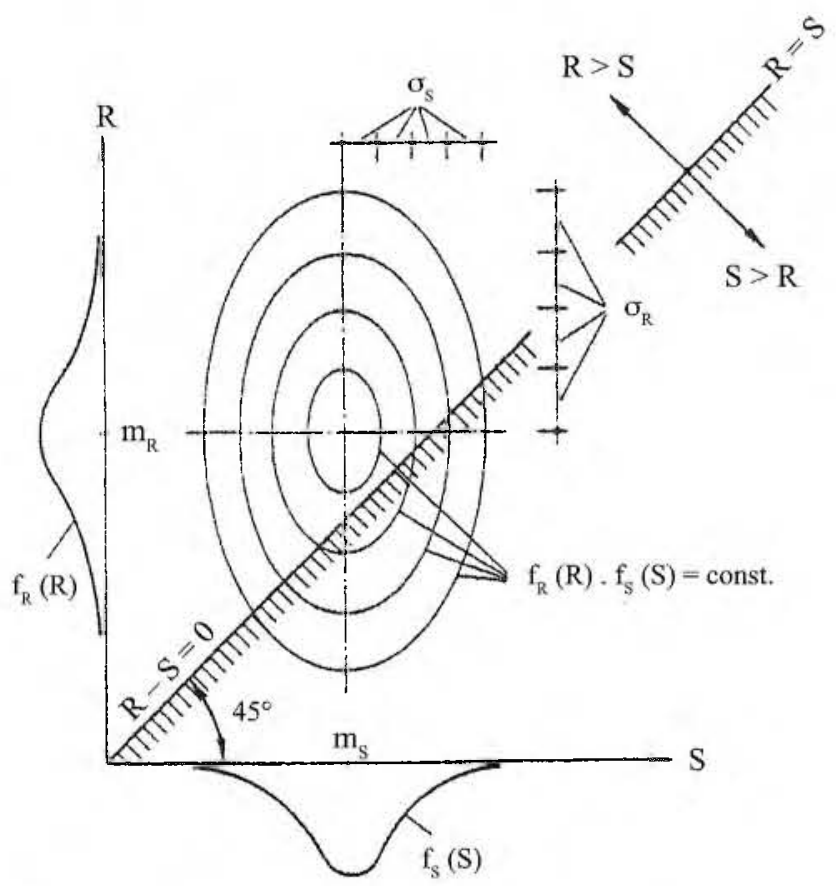

FIG. 2 Représentation de l'équation d'état limite et des densités de probabilité dans le système d'axes originel.

Representation of the limit state equilibrium and densities of probability in the original axis system.

Pour déterminer l'indice de fiabilité $\beta$, toutes les variables aléatoires non normales (ici $\mathrm{R}$ et $\mathrm{S}$ ) doivent être normalisées en passant dans le plan réduit $(\mathrm{m}=0$, $\sigma=1$ ) par la relation:

$$
\mathrm{U}=\frac{\left(\mathrm{x}_{\mathrm{i}}-\mathrm{m}_{\mathrm{x}_{\mathrm{i}}}\right)}{\sigma_{\mathrm{x}_{\mathrm{i}}}}
$$

où $U$ est la variable aléatoire réduite ; $X_{i}$, la variable aléatoire ; $m_{x_{j}}$, la valeur moyenne de la variable aléatoire et $\sigma_{X_{1}}, l^{\prime}$ écart-type de la variable aléatoire.
A travers cette transformation, les ellipses deviennent des cercles (Fig. 3).

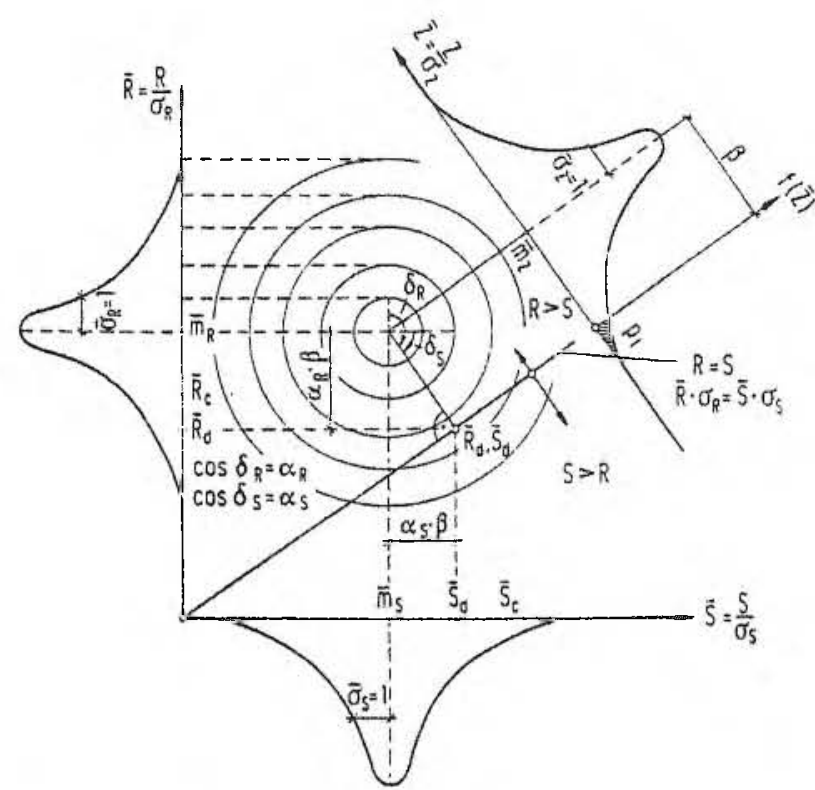

FIG. 3 Indice de fiabilité et valeurs de calcul dans le plan standardisé ou réduit.

Reliability index and computation values in the standard or reduced plane.

L'équation d'état limite, qui est en réalité dans le cas général souvent courbe, est remplacée approximativement par une droite tangentielle (Späthe, 1987). D'après la définition de Hasofer/Lind (1974), citée par Späthe (1987), l'indice de fiabilité $\beta$ est égal à la plus courte distance entre le milieu du cercle, courbes d'équiprobabilité, et la droite tangentielle approximative de l'équation d'état limite (Späthe, 1987 ; Ditlevsen, 1982 ; JCSS, 2001). Si on projette maintenant les courbes d'équiprobabilité sur un plan perpendiculaire à la droite tangentielle, on obtient une courbe de distribution qui correspond à la densité de probabilité de la marge de sécurité $Z$ (Fig. 3). De cette projection, on déduit l'indice de fiabilité $\beta$ et la zone négative correspond à la probabilité de rupture ou de ruine $\mathrm{P}_{\mathrm{f}}$.

Il existe une relation mathématique entre la probabilité de rupture $P_{f}$ et l'indice de fiabilité $\beta$

$$
\mathrm{P}_{\mathrm{f}}=\Phi(-\beta)=\frac{1}{\sqrt{2 \pi}} \cdot \int_{-\infty}^{0} \exp \left[-\frac{1}{2}\left(\frac{\mathrm{z}}{\sigma_{\mathrm{z}}}-\beta\right)^{2}\right] \cdot \mathrm{d}\left(\frac{\mathrm{z}}{\sigma_{\mathrm{z}}}\right)
$$

avec $\Phi$ ( ) la fonction de distribution normale standardisée ou réduite. Des exemples numériques de cette dernière relation sont regroupés dans le tableau I.

tableau I Relation entre l'indice de fiabilité $\beta$ 's et la probabilité de rupture Pf (d'après l'équation 8). Relation between reliability index $\beta$ and probability of failure $P_{f}$ (after equation 8).

\begin{tabular}{lcccccc}
\hline$\beta[-]$ & 5,2 & 4,7 & 4,2 & 3 & 2,5 & 2 \\
$P_{f}[-]$ & $\approx 10^{-7}$ & $\approx 10^{-6}$ & $\approx 10^{-5}$ & $\approx 10^{-3}$ & $\approx 5.10^{-3}$ & $\approx 10^{-2}$
\end{tabular}

L'indice de fiabilité $\beta$ déterminé par cette méthode correspond à la sécurité minimale. La perpendiculaire abaissée du milieu du cercle donne par intersection avec la droite tangentielle le point le plus dangereux sur la courbe de l'équation d'état limite qui présente la plus grande probabilité de ruine (Fig. 3). Ce point est 
appelé le « point de calcul » et ses coordonnés $\mathrm{R}_{\mathrm{d}}$ et $\mathrm{S}_{\mathrm{d}}$ sont appelées « valeurs de calcul » (Belabed, 1996) :

$$
R_{d}=m_{R}-\beta \cdot \alpha_{R} \cdot \sigma_{R} ; S_{d}=m_{s}+\beta \cdot \alpha_{s} \cdot \sigma_{s}
$$

où l'indice ( $\mathrm{d}$ ) signifie design (valeur de calcul) et

$$
\alpha_{\mathrm{R}}=\frac{\sigma_{\mathrm{R}}}{\sqrt{\sigma_{\mathrm{R}}^{2}+\sigma_{\mathrm{S}}^{2}}} ; \alpha_{\mathrm{S}}=\frac{\sigma_{\mathrm{S}}}{\sqrt{\sigma_{\mathrm{R}}^{2}+\sigma_{\mathrm{S}}^{2}}}
$$

sont les facteurs de sensibilité ou les cosinus directeurs. Les facteurs de sensibilité $\alpha_{i}$ peuvent prendre des valeurs entre -1 et +1 . Plus le facteur de sensibilité $\left|\alpha_{i}\right|$ se rapproche de l'unité, plus la contribution à la probabilité de ruine d'un paramètre de base est importante (Nottrodt, 1990 ; Belabed, 1996). La condition suivante doit être vérifiée : $\sum_{i=1}^{n} \alpha_{i}^{2}=1$.

Les résistances et les sollicitations de calcul sont déterminées pratiquement en introduisant les coefficients partiels de sécurité, qui permettent de les calculer simplement à partir des valeurs moyennes $m_{R}$ et $\mathrm{m}_{\mathrm{S}}$ :

$$
R_{d}=\frac{m_{B}}{\gamma_{R}} ; S_{d}=\gamma_{S} \cdot m_{S}
$$

où $\gamma$ et $\gamma$ sont les facteurs partiels de sécurité respectivement de sollicitation et de résistance.

Pour vérifier la sécurité d’une structure quelconque (par exemple : une fondation superficielle), la relation suivante doit donc être satisfaite :

$$
\mathrm{R}_{\mathrm{d}} \geq \mathrm{S}_{\mathrm{d}}
$$

A partir des équations (9) et (11), on obtient les facteurs partiels de sécurité :

$$
\begin{array}{lll}
\gamma_{S}=1+\beta \cdot \alpha_{S} \cdot V_{S} & \text { avec } & V_{S}=\frac{\sigma_{S}}{m_{S}} \\
\gamma_{R}=\frac{1}{1-\beta \cdot \alpha_{R} \cdot V_{R}} & \text { avec } & V_{R}=\frac{\sigma_{R}}{m_{R}}
\end{array}
$$

où $V_{R}$ et $V_{S}$ sont des coefficients de variation respectivement de la résistance $R$ et de la sollicitation $S$.

L'analyse des équations (9) à (14) montre l'influence du coefficient de variation et du type de distribution de la densité de probabilité $(\sigma, \mathrm{m})$ sur le calcul probabiliste de la sécurité (valeurs de calcul des paramètres et facteurs partiels de sécurité). La détermination de ces paramètres statistiques doit se faire aussi minutieusement que possible, ce qui est souvent difficile en mécanique des sols à cause de l'insuffisance des données géotechniques.

En réalité, dans le cas général, le nombre de variables aléatoires est supérieur à deux, ce qui rend le calcul graphique et manuel de $\beta$ très complexe et pénible. Le point de calcul est recherché de manière itérative en utilisant des algorithmes de calcul tels cue l'algorithme de Späthe (1987) et l'algorithme de Rackwitz/Fießler (Fießler et al., 1976 ; Rackwitz, 1978). Ces algorithmes sont intégrés dans le logiciel ZUVAN (Belabed, 1996), utilisé dans ce travail.

Les vérifications de fiabilité des ouvrages sont généralement effectuées suivant deux méthodes : la méthode probabiliste ou la méthode semi-probabiliste.

Avec les méthodes probabilistes, on peut calculer les facteurs partiels de sécurité nécessaires pour la vérification de la sécurité (fiabilité) d'après la méthode semi-probabiliste. La méthode probabiliste est inconnue pour l'ingénieur praticien et nécessite des connaissances approfondies en probabilité, ce qui la rend pra- tiquement inutilisable. En revanche, dans la méthode semi-probabiliste, le praticien n'a pas besoin d'avoir des connaissances approfondies en probabilité puisque les facteurs partiels de sécurité lui sont donnés directement par des normes spécifiques. Dans notre travail. l'étude de lá stabilité des fondations superficielles vis-à-vis du poinçonnement a été effectuée d'après la méthode semi-probabiliste.

\section{3}

\section{Modèles mécaniques}

Pour la modélisation mécanique de la rupture des fondations superficielles par poinçonnement, on trouve dans la littérature plusieurs méthodes qui sont en général inspirées de la théorie classique de Prandtl : formation d'un cône rigide solidaire de la fondation (Fig. 4). Dans ce travail, on a étudié deux types de fondation superficielle : la semelle filante et la semelle carrée. On suppose que :

- la semelle repose sur un massif homogène horizontal ;

- l'effet des contraintes de cisaillement au contact solfondation est négligé ;

- le sol situé au-dessus de l'horizontale AA' de la base de la fondation agit comme une surcharge verticale constante d'intensité $\gamma \mathrm{D}$ (Figs. 4 et 6) ;

- aucune nappe d'eau n'est présente.

\section{1}

\section{Semelle fillante}

On considère le cas d'une semelle filante de largeur B enterrée dans le massif à une profondeur D (Figs. 4 et 6 ). Trois cas de charge ont été analysés.

\section{Charge verticale centrée}

Le modèle mécanique classique de rupture est représenté sur la figure 4. L'équation d'étal limite générale donnant la contrainte limite de rupture s'écrit:

$$
q_{L}=\frac{1}{2} \cdot \gamma \cdot B \cdot N_{\gamma}+\gamma \cdot D \cdot N_{q}+c^{\prime} \cdot N_{c}
$$

où $\gamma$ est le poids volumique du sol et $c^{\prime}$, la cohésion effective du sol.

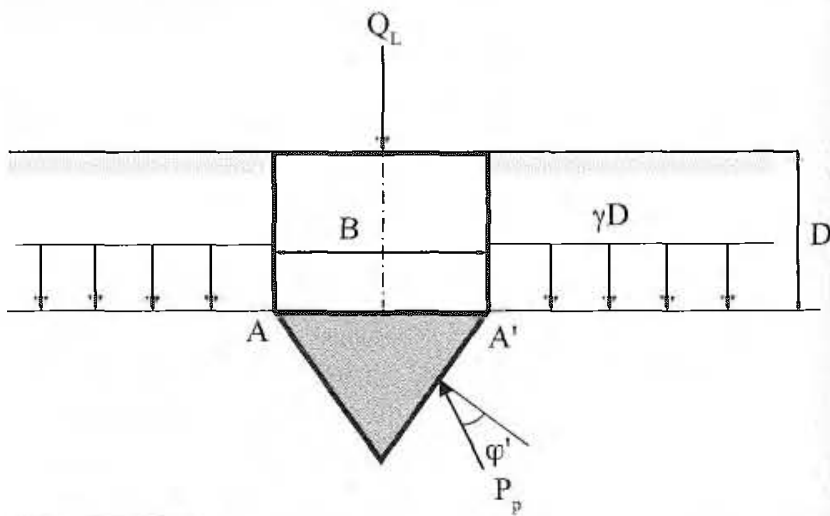

FIG.4. Équilibre des terres sous la fondation (charge verticale centrée).

Equilibrium of earth mass under the foundation (vertical centered load) 
TABLEAU II Facteurs de capacité portante.

Bearing capacity factors.

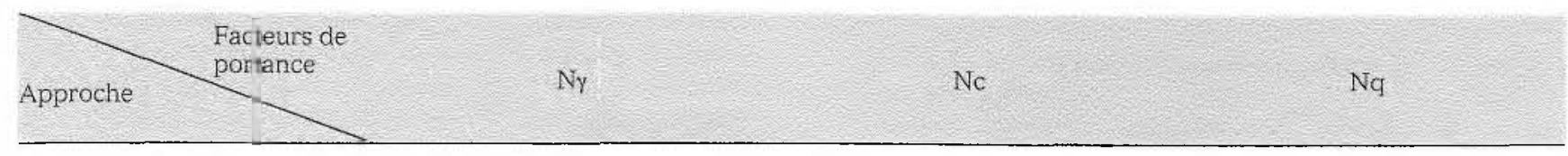

DTU 13.12 (1988)

DIN 1054 (1976 et 2005)

Eurocode 7 (2004)
$1,85\left(\mathrm{~N}_{\mathrm{q}}-1\right) \operatorname{tg} \varphi^{\prime}$

$2\left(N_{q}-1\right) \operatorname{tg} \varphi^{\prime}$

$2\left(N_{4}-1\right) \operatorname{tg} \varphi^{\prime}$ $\operatorname{cotg} \varphi^{\prime}\left(\mathrm{N}_{q}-1\right)$

$\operatorname{tg}^{2}\left(\frac{\pi}{4}+\frac{\varphi^{\prime}}{2}\right) e^{\pi \operatorname{tg} \varphi^{\prime}}$

$\operatorname{cotg} \varphi^{\prime}\left(\mathrm{N}_{q}-1\right)$

$\operatorname{tg}^{2}\left(\frac{\pi}{4}+\frac{\varphi^{\prime}}{2}\right) \mathrm{e}^{\pi \operatorname{tg} \varphi^{\prime}}$

$\operatorname{cotg} \varphi^{\prime}\left(\mathrm{N}_{4}-1\right)$ $\operatorname{tg}^{2}\left(\frac{\pi}{4}+\frac{\varphi^{\prime}}{2}\right) e^{\pi \operatorname{tg} \varphi^{\prime}}$
$N_{,} N_{q}$ et $N_{c}$ sont les facteurs de capacité portante dépendant de l'angle de frottement interne $\varphi^{\prime}$. Le tableau II regroupe les formules de calcul de ces trois facteurs suivant l'approche française DTU 13.12 (1988) et l'approche allemande DIN 1054 (1976 et 2005), ainsi que pour l'Eurocode 7 (2004).

\section{Charge verticale excentrée}

Des essais sur modèles réduits ont montré qu'en milieu pulvérulent le coin qui se crée sous la fondation à la rupture garde la même forme que pour une charge verticale centrée, mais que ses dimensions se réduisent de manière à ce que la ligne d'action de la charge et l'axe de symétrie du coin coincident. Dans le cas d'une semelle filante supportant une charge verticale excentrée suivant B (Fig. 5), Meyerhof (1953), cité par Costet et Sanglerat (1983) et Frank (2003), a proposé d'attribuer à cette semelle une largeur fictive centrée sur la charge $\mathrm{B}^{\prime}=\mathrm{B}-2 \mathrm{e}$, où e désigne l'excentricité de la charge.

L'équation d'état limite générale donnant la charge limite de rupture par mètre de semelle est :

$$
Q_{L}=q_{L} \cdot B^{\prime}=B^{\prime}\left[\frac{1}{2} \cdot \gamma \cdot B^{\prime} \cdot N_{y}+\gamma \cdot D \cdot N_{q}+c^{\prime} \cdot N_{c}\right]
$$
ches.

Cette équation est valable pour toutes les appro-

TABLEAU III Coefficients correcteurs pour une charge inclinée.

Correction coefficients for inclined load.

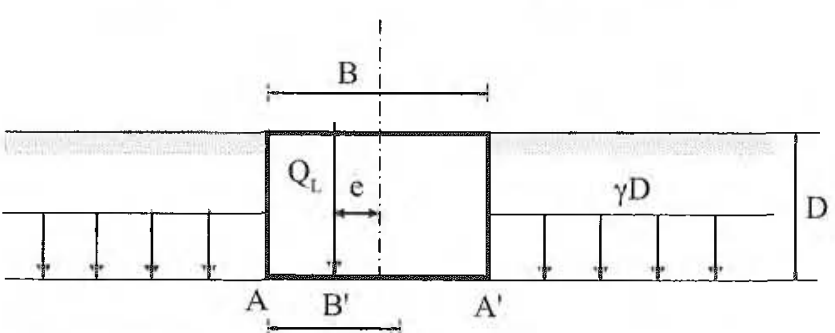

FIG. 5 Charge verticale excentrée. Eccentric verlical load.

\section{Charge centrée inclinée}

L'inclinaison de la charge diminue fortement la capacité portante des fondations (Fig. 6). Meyerhof (1953), cité par Costet et Sanglerat (1983) et Frank (2003), a également résolu le problème de manière approchée en introduisant des coefficients correcteurs

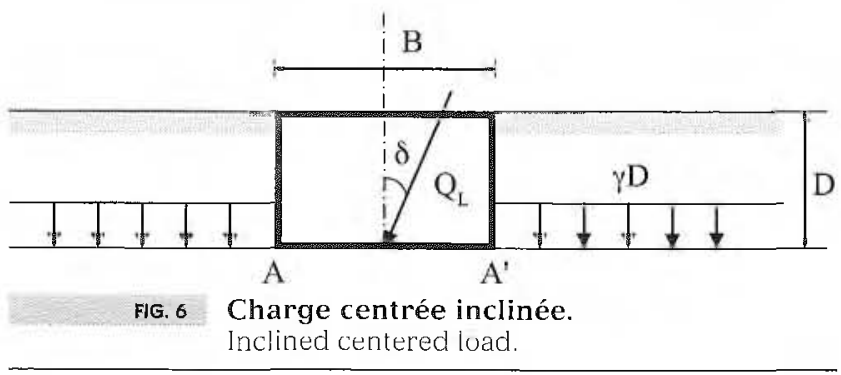

\begin{tabular}{|c|c|c|c|}
\hline Approche & & ${ }^{t} q$ & $i_{\gamma}$ \\
\hline DTU 13.12 (1988) & $\left(1-\frac{2 \delta}{\pi}\right)^{2}$ & $\left(1-\frac{2 \delta}{\pi}\right)^{2}$ & $\left(1-\frac{\delta}{\varphi^{\prime}}\right)^{2}$ \\
\hline DIN 1054 (1976) & $\left(\mathrm{i}_{\mathrm{q}} \mathrm{N}_{\mathrm{q}}-1\right) /\left(\mathrm{N}_{\mathrm{q}}-1\right)$ & $\left(1-0,7 \mathrm{H} /\left(\mathrm{V}+\mathrm{B}^{\prime} \mathrm{c}^{\prime} \operatorname{cotg} \varphi^{\prime}\right)\right)^{3}$ & $\left(1-\mathrm{H} /\left(\mathrm{V}+\mathrm{B}^{\prime} \mathrm{c}^{\prime} \operatorname{cotg} \varphi^{\prime}\right)\right)^{3}$ \\
\hline DIN 1054 (2005) & $\left(\mathrm{i}_{\mathrm{q}} \mathrm{N}_{\mathrm{q}}-1\right) /\left(\mathrm{N}_{\mathrm{q}}-1\right)$ & $(1-\operatorname{tg} \delta)^{2}$ & $(1-\operatorname{tg} \delta)^{3}$ \\
\hline Eurocode 7 (2004) & $\left(\mathrm{i}_{\mathrm{q}} \mathrm{N}_{\mathrm{q}}-1\right) /\left(\mathrm{N}_{\mathrm{q}}-1\right)$ & $\left(1-H /\left(V+B^{\prime} c^{\prime} \operatorname{cotg} \varphi^{\prime}\right)\right)^{2}$ & $\left(1-\mathrm{H} /\left(\mathrm{V}+\mathrm{B}^{\prime} \mathrm{c}^{\prime} \operatorname{cotg} \varphi^{\prime}\right)\right)^{3}$ \\
\hline
\end{tabular}


d'inclinaison $i_{\gamma,} i_{c}$ et $i_{c}$ dans les trois termes de l'expression de la pression de rupture (16)

L'équation d'état limite générale donnant la contrainte limite de rupture est ainsi :

$$
q_{L}=\frac{1}{2} \cdot \gamma \cdot B \cdot N_{\gamma} \cdot i_{\gamma}+\gamma \cdot D \cdot N_{q} \cdot i_{q}+c^{\prime} \cdot N_{c} \cdot i_{c}
$$

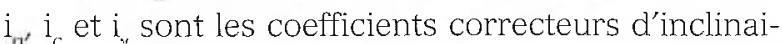
son dépendant de l'inclinaison de la charge $\delta$ (Fig. 6), de l'angle de frottement interne $\varphi^{\prime}$ et de la cohésion c'. Le tableau III fournit les formules de ces trois coefficients pour les différentes approches de calcul : $\delta$ est l'angle d'inclinaison de la charge par rapport à la verticale ; $\mathrm{H}$ et $\mathrm{V}$, les composantes horizontale et verticale de la charge extérieure $\mathrm{Q}$ par mètre de semelle centrée et inclinée de l'angle $\delta$ par rapport à la verticale (Fig. 6).

\section{2}

\section{Semelle carrée}

Pour tenir compte de la forme géométrique de la semelle (largeur B et longueur L), on utilise la formule donnant la capacité portante de la semelle filante (éq. 15) en affectant chacun des trois facteurs de capacité portante de coefficients correcteurs appelés facteurs de forme $S_{\alpha}, S_{c}$ et $S_{\gamma}$. Ces facteurs sont établis de manière empirique à partir de résultats d'essais de laboratoire et de constatations faites sur la capacité portante de semelles réelles. Le tableau IV regroupe les formules de ces trois facteurs de forme pour différentes approches de calcul (pour une semelle carrée faire B = L).

Les mêmes cas de chargement que pour les semelles filantes ont été étudiés pour les semelles carrées. On constate une bonne concordance entre l'Eurocode 7 (2004) et la norme allemande DIN 1054 (1976 et 2005) dans les formules de calcul des différents facteurs intervenant dans l'estimation de la capacité portante. En revanche, le règlement français DTU 13.12 (1988) propose généralement des formules différentes (voir tableaux II, III et IV).

\section{Études paramétriques}

\section{1}

\section{Introduction}

L'étude semi -probabiliste de la capacité portante des fondations superficielles est effectuée en deux étapes :

- dimensionner la semelle en déterminant sa largeur minimale d'après différentes approches pour différents cas ;

- évaluer la sécurité correspondante en calculant la probabilité de rupture ou l'indice de tiabilité.

Les études paramétriques ont été effectuées pour une semelle de largeur B et de longueur L, encastrée d'une profondeur $\mathrm{D}=1.5 \mathrm{~m}$ dans un sol homogène de poids volumique égal à $20 \mathrm{kN} / \mathrm{m}^{3}$ (tableau V). Le poids volumique du béton est pris égal à $24 \mathrm{kN} / \mathrm{m}^{3}$.

Différents cas ont été étudiés en faisant varier les paramètres de calcul suivants :

- Caractéristiques du sol (sol cohérent et sol pulvérulent) ;

- types de fondation superficielle (semelle filante, semelle carrée) :

- intensité et point d'application des charges (charge verticale centrée, charge verticale excentrée et charge inclinée). On suppose qu'aucune charge variable n'est appliquée sur la semelle $\left(\mathrm{N}_{0}=0\right)$.

tABLEAU IV Facteurs de forme pour semelles rectangulaire et carrée (B largeur et $\mathrm{I}$. longueur d'une semelle). Shape coefficients for rectangular and square shallow foundation (width $B$ and length $\mathrm{L}$ ).

\begin{tabular}{|c|c|c|c|}
\hline Approche & $\mathrm{S}_{\mathrm{C}}$ & $\mathrm{s}_{\mathrm{q}}$ & $S_{y}$ \\
\hline DTU 13.12 (1988) & $1+0,2 \frac{\mathrm{B}}{\mathrm{L}}$ & 1 & $1-0,2 \frac{B}{L}$ \\
\hline DIN 1054 (1976 et 2005) & $\frac{\left(1+\frac{B}{L} \sin \varphi^{\prime}\right) N_{q}-1}{N_{q}-1}$ & $1+\frac{B}{L} \sin \varphi^{\prime}$ & $1-0,3 \frac{\mathrm{B}}{\mathrm{L}}$ \\
\hline Eurocode 7 (2004) & $\frac{\left(1+\frac{B}{L} \sin \varphi^{\prime}\right) N_{q}-1}{N_{q}-1}$ & $1+\frac{B}{L} \sin \varphi^{\prime}$ & $1-0,3 \frac{B}{L}$ \\
\hline
\end{tabular}

tABLEAU v Données statistiques des variables aléatoires.

Statistical data for random variables.

\begin{tabular}{lccc} 
Paramètre & Type de distribution & Coefficient de variation & Valeur caractéristique \\
\hline Angle de frottement interne $\varphi^{\prime}$ [degrés] & Log-normale & $10 \%$ & 20 à 40 \\
Cohésion $c^{\prime}[\mathrm{kPa}]$ & Log-normale & $25 \%$ & 0 à 35 \\
Poids volumique du sol $\gamma\left[\mathrm{kN} / \mathrm{m}^{3}\right]$ & Normale & $5 \%$ & 20 \\
Charge extérieure permanente $\mathrm{Q}[\mathrm{kN} / \mathrm{ml}]$ & Normale & $10 \%$ & 290 à 800 \\
Inclinaison de la charge $\delta[$ degrés] & Normale & $10 \%$ & 10 \\
\hline
\end{tabular}


La valeur caractéristique d'un paramètre doit être déterminée avec prudence en tenant compte entre autres de la variabilité naturelle, des incertitudes d'échantillonnage et du volume de sol concerné par le mécanisme de rupture (Eurocode 7, 2004). En géotechnique, le nombre d'essais in situ et au laboratoire est généralement insuffisant pour évaluer rigoureusement la valeur caractéristique d'un paramètre de sol à partir de considérations statistiques. Ce fait conduit à recourir à une banque de données pour couvrir le manque d'informations. Le traitement de ces données se fait au moyen de méthodes statistiques telles que celle de Bayes, Student et Schneider (Baguelin et Kovarik, 2000 ; Orr, 2000 ; Favre, 2004).

Le coefficient de variation, la valeur moyenne et l'écart-type (type de distribution de la densité de probabilité) influent grandement sur la valeur caractéristique et la valeur de calcul d'un paramètre (éq. 9 et 14). La valeur caractéristique d'un paramètre $\mathrm{X}$ peut être calculée par la relation suivante

$$
\mathrm{X}_{\mathrm{k} i}=\mathrm{m}_{\mathrm{i}}+\mathrm{K}_{\mathrm{i}}(\mathrm{n}) \cdot \sigma_{\mathrm{i}}=\mathrm{m}_{\mathrm{l}} \cdot\left(1+\mathrm{K}_{\mathrm{l}}(\mathrm{n}) \cdot \mathrm{V}_{\mathrm{i}}\right)
$$

où $V_{i}$ est le coefficient de variation; $m_{j}$, la valeur moyenne ; $\sigma_{i}$, l'écart-type ; $n$, le nombre d'essais et $\mathrm{K}_{\mathrm{i}}$ un coefficient déterminé en considérant le fractile à $5 \%$ pour les résistances et le fractile à $95 \%$ pour les actions. A titre d'exemple, pour une loi normale de Gauss, ceci correspond à $K= \pm 1,65$. Ces valeurs caractéristiques sont affectées encore de coefficients pondérateurs (Breitschaft et Hanisch, 1978). Baguelin et Kovarik (2000) font appel à la loi de Student pour définir la valeur caractéristique et mettent en évidence l'influence de la variation spatiale (auto-corrélation) du paramètre sur sa valeur caractéristique.

\section{2}

\section{Modèle stochastique}

Nottrodt (1990) a pu montrer, dans sa thèse de doctorat, qu'il y a une relation inversement proportionnelle entre les paramètres de cisaillement effectifs du sol $\left(\varphi^{\prime}\right.$ et $\left.c^{\prime}\right)$ : les petites valeurs de $\varphi^{\prime}$ correspondent à des grandes valeurs de $c^{\prime}$ et vice versa. Cette relation est exprimée par un coefficient de corrélation négatif élevé. Sur la base de ces résultats et de ceux de Genske et Walz (1987) et Belabed (1996), des calculs ont été réalisés avec des combinaisons de paramètres de cisaillement considérées comme relativement typiques en mécanique des sols (tableau V). De plus, on néglige dans ce travail l'effet de la corrélation entre variables et de l'autocorrélation, et on suppose que les valeurs caractéristiques des paramètres ont été déterminées avec prudence conformément aux recommandations de l'Eurocode 7 (2004).

En se basant sur les études expérimentales et statistiques effectuées entre autres par Nottrodt (1990), Soos (1982) et Pottharst (1982) sur plusieurs échantillons de sols et les études théoriques réalisées par Gäßler (1987) et Genske et Walz (1987), ainsi que les propositions de Meyerhof citées par Favre (2004) (tableau IV, p. 187) et celles d'Abramson et al. (2001), on a proposé les distributions aléatoires résumées dans le tableau $V$ pour nos études paramétriques. Pour la cohésion et l'angle de frottement interne, on admet qu'ils suivent tous les deux une loi log-normale, ce qui est physiquement relativement exact, car les valeurs négatives sont ainsi exclues. On sait que l'angle de frottement interne est moins dispersé que la cohésion. On adopte alors des coefficients de variation moyens de $10 \%$ pour l'angle de frottement interne $\varphi^{\prime}$ et de $25 \%$ pour la cohésion $C^{\prime}$. De plus, on admet pour l'angle de frottement interne un seuil de troncature égal à $\varphi^{\prime} / 2$ (distribution tronquée), ce qui évite d'avoir des valeurs de $\varphi^{\prime}$ proches à 0 . Cela influe légèrement sur le niveau de sécurité (Genske et Walz, 1987 ; Belabed, 1996). Les paramètres géométriques (encastrement de la semelle D, largeur de la semelle B, etc.) présentent en général une dispersion négligeable par rapport à celle des paramètres de cisaillement du sol et des charges (Nottrodt, 1990 ; Gäßler, 1987). C'est pourquoi on les considère comme des paramètres déterministes. Les calculs probabilistes effectués par Nottrodt (1990), Genske et Walz (1987) et Gäßler (1987) ont montré, d'une part, que le facteur de sensibilité $\alpha_{j}$ pour le poids volumique du sol $\gamma$ est très petit en comparaison des facteurs de sensibilité de l'angle de frottement interne et de la cohésion et, d'autre part, que les facteurs partiels de sécurité dérivés pour le poids volumique du sol sont très proches de 1 . Pour cette raison, on admet pour le poids volumique du sol un facteur partiel de sécurité égal à 1 et un coefficient de variation de $5 \%$. On admet également que la charge extérieure permanente $Q$, son inclinaison $\delta$ et le poids volumique du sol suivent une loi normale (Genske et Walz, 1987 ; Nottrodt, 1990 ; Vrouwenvelder, 2002 ; Sørensen, 2002).

\section{5}

\section{Dimensionnement des semelles et analyse de la fiabilité}

On détermine la largeur B minimale pour différents types de semelles filantes et carrées vérifiant l'équilibre du système suivant deux approches, approche déterministe et approche semi-probabiliste (Eurocode 7 , 2004).

Dans le cas général, la justification de la structure est envisagée pour des actions permanentes, variables et transitoires : situations durables, transitoires et accidentelles. Les combinaisons de charge de calcul à I’ELU sont :

- combinaisons fondamentales correspondant à des situations de calcul durables et transitoires ;

- combinaisons accidentelles correspondant à des situations de calcul rares (exemple: séisme).

On ne parle, dans cet article, que des combinaisons fondamentales à l'exclusion des ELU situations accidentelles.

\section{1}

\section{Approche déterministe}

La sécurité est exprimée par un facteur global de sécurité $F_{S}$ qui est égal en général au rapport de la résistance $\mathrm{R}$ sur la sollicitation $\mathrm{S}$ :

$$
\frac{\mathrm{R}}{\mathrm{S}} \geq \mathrm{F}_{\mathrm{S}}
$$

Les forces et les paramètres du sol ne sont pas pondérés. 


\section{Approche française}

La sécurité vis-à-vis du poinçonnement d'une fondation superficielle est exprimée par l'inéquation suivante d'après le DTU 13-12 (1988) :

$$
\mathrm{q}_{\mathrm{réel}} \leq \mathrm{q}_{\mathrm{ad}}
$$

où $q_{\text {réel }}$ est la contrainte réelle non pondérée appliquée à la fondation et $\mathrm{q}_{\mathrm{ad}}$ la contrainte admissible.

La contrainte réelle se calcule d'après la figure 4 comme suit :

$$
\mathrm{q}_{\text {réel }}=\frac{\mathrm{Q}+\gamma_{\text {béton }} \cdot \text { B.D.L }}{\text { B.L }}
$$

Cette contrainte représente l'action permanente sur la fondation.

La contrainte admissible est la contrainte limite que peut supporter la fondation divisée par un coefficient (facteur) global de sécurité désigné par $F_{S}$, pris égal à 2. Le coefficient de sécurité n'est pas appliqué sur le poids des terres :

$$
q_{a d}=\gamma \cdot D+\frac{\gamma \cdot \frac{B}{2} \cdot N_{\gamma}+\gamma \cdot D \cdot\left(N_{q}-1\right)+c^{\prime} \cdot N_{c}}{F_{s}}
$$

Dans les développements qui suivent, cette approche est nommée approche DTU 13.12.

\section{- Exemple numérique}

Soit une semelle filante soumise à une charge permanente verticale centrée $(\mathrm{Q}=290 \mathrm{kN} / \mathrm{m})$. On suppose une charge (action) variable nulle. Les paramètres de comportement du sol sont : $\varphi^{\prime}=30$ degrés et $\mathrm{c}^{\prime}=10 \mathrm{kPa}$.

La contrainte réelle exercée sur la semelle filante est calculée d'après l'équation (21): $q_{\text {réel }}=\frac{290+36 \mathrm{~B}}{\mathrm{~B}}$.

Les facteurs de portance sont calculés d'après le tableau II pour $\varphi^{\prime}=30$ degrés. On obtient : $\mathrm{N}_{\mathrm{c}}=18,4$; $\mathrm{N}_{\mathrm{y}}=18,59$ et $\mathrm{N}_{\mathrm{c}}=30,14$. Pour $\mathrm{F}_{\mathrm{s}}=2$, on peut calculer la contrainte admissible d'après l'équation (22) :

$$
q_{a d}=92,95 B+441,7
$$

En remplaçant les termes calculés $q_{\text {réel }}$ et $q_{a d}$ dans l'inéquation (20), on obtient une inéquation du deuxième degré : $92,95 \mathrm{~B}^{2}+405,7 \mathrm{~B}-290>0$

dont la solution fournit la largeur minimale $\mathrm{B}=0,63 \mathrm{~m}$ (voir tableau VIII, colonne 3 , ligne 7 ).

\section{Approche traditionnelle allemande}

La vérification de la stabilité d'une fondation superficielle vis-à-vis du poinçonnement est effectuée par l'inéquation suivante d'après la norme allemande ancienne DIN 1054 (1976) :

$$
\frac{V_{b}}{V} \geq F_{S}
$$

avec $V_{b}=q_{L} \cdot B$

$\mathrm{B}$ est la surface de la semelle; $\mathrm{F}_{\mathrm{S}^{\prime}}$ le facteur global de sécurité pris égal $2 ; V_{b}$, la charge limite que peut supporter la fondation; $V$, les charges extérieures appliquées sur la fondation y compris le poids propre.

Dans les calculs qui suivent, cette approche est nommée approche DIN $1054\left(\mathrm{~F}_{\mathrm{S}}=2\right)$.

\section{- Exemple numérique}

On reprend le même exemple que précédemment.

D’après la figure 4 , la charge extérieure verticale totale est égale à

$\mathrm{V}=\mathrm{Q}+\gamma_{\text {bét nn }}$ D.B.1 $=290+36 \mathrm{~B}$

D'après le tableau II et pour $\varphi^{\prime}=30$ degrés : $\mathrm{N}_{c}=18,4 ; \mathrm{N}_{\mu}=20,09$ et $\mathrm{N}_{c}=30,14$. Ces valeurs sont reportées dan's l'équation (15) pour calculer $q_{L}$.

On calcule ensuite la charge limite : $V_{D}=q_{L} \cdot B$ $=(200,9 \mathrm{~B}+853,4) \cdot \mathrm{B}$.

Pour $F_{S}=2$ et en remplaçant les expressions de $V$ et $V_{b}$ dans l'inéquation (23), on obtient l'inéquation suivante : $200,9 \mathrm{~B}^{2}-781,4 \mathrm{~B}-580 \geq 0$ qui fournit la largeur minimale de la fondation $\mathrm{B}=0,64 \mathrm{~m}$ (voir tableau VIII, colonne 2, ligne 7).

\section{2}

\section{Approche semi-probabiliste}

\section{Généralités}

D'après l'Eurocode 7 (20()4), on distingue deux états limites : l'état limite ultime et l'état limite de service. Les états limites ultimes suivants doivent être vérifiés lorsqu'ils sont pertinents :

- EQU (Équilibre) : perte d'équilibre statique de la structure ou du terrain, dans lesquels les résistances des matériaux (de la structure ou du terrain) n'apportent pas de contribution significative à la résistance (exemple : renversement) ;

STR (Structure) : rupture interne ou déformation excessive de la structure ou d'éléments de la structure, dans lesquels la résistance des matériaux de la structure contribue signifïcativement à la résistance (exemple : fondations, ancragesi;

- GEO (Géotechnique) : rupture ou déformation excessive du terrain, dans lequel la résistance des sols ou des roches contribue de façon significative à la résistance (exemple : glissement des pentes, poinçonnement) ;

- UPL (Soulèvement) : rupture par soulèvement dû à des forces verticales (exemple : poussée d'Archimèdel ;

- HYD (Hydraulique) : rupture causée par les gradients hydrauliques dans le terrain (exemple : érosion interne, Renard).

\section{Principe de la vérification de la résistance pour les états limites de la structure et du terrain}

Pour les états limites de rupture ou de déformation excessive d'un élément de structure ou du terrain (STR et GEOJ, les sollicitations sont majorées et les résistances minorées avec des facteurs partiels de sécurité de telle sorte que

$$
\mathrm{R}_{\mathrm{c}}-\mathrm{N}_{\mathrm{c}} \geq 0
$$

où $\mathrm{R}_{1}$ est la résistance de calcul ou capacité portante de calcul et $\mathrm{N}_{\text {, }}$, la sollicitation (charge) de calcul appliquée à la fondation tenant compte d'éventuels coeflicients pondérateurs des charges (généralement supérieurs à 1).

Pour le calcul de la capacité portante (résistance) de calcul, on distingue deux cas. 


\section{- Cas 1}

Si les facteurs partiels de sécurité sont appliqués sur les forces, la capacité portante de calcul est calculée comme suit

$$
R_{d}=\frac{Q_{L}}{\gamma_{R}} \text { avec } Q_{L}=q_{L} B
$$

où $Q_{L}$ est la capacité portante caractéristique calculée avec les paramètres caractéristiques du sol et $\gamma_{R^{\prime}}$ le facteur partiel de sécurité appliqué sur la résistance (capacité portante caractéristique), voir tableaux VI et VII.

\section{- Cas 2}

Si les facteurs partiels de sécurité sont appliqués sur les paramètres du sol $\left(\gamma, c^{\prime}, \varphi^{\prime}\right)$, la capacité portante de calcul est calculée avec les paramètres de calcul du sol :

$$
R_{d}=Q_{L}\left(\varphi_{d^{\prime}}^{\prime}, C_{d^{\prime}}^{\prime} \gamma_{d}\right)=q_{L}\left(\varphi^{\prime} d^{\prime} C_{d^{\prime}}^{\prime} \gamma_{d}\right) \cdot B
$$

$\operatorname{avec} \tan \varphi_{\mathrm{d}}^{\prime}=\frac{\tan \varphi_{\mathrm{k}}^{\prime}}{\gamma_{\varphi^{\prime}}} ; \quad c_{\mathrm{d}}^{\prime}=\frac{c_{\mathrm{k}}^{\prime}}{\gamma_{\mathrm{c}^{\prime}}}$ et $\gamma_{\mathrm{d}}=\gamma_{\mathrm{k}}$

où $\gamma_{\varphi^{\prime}}$ est le facteur partiel de sécurité appliqué sur l'angle de frottement interne effectif et $\gamma_{C^{\prime}}$ le facteur partiel de sécurité appliqué sur la cohésion effective. L'indice $(\mathrm{k})$ signifie valeur caractéristique et l'indice ( $\mathrm{d}$ ) valeur de calcul.

La sollicitation ou la charge de calcul est estimée par la combinaison

$$
\mathrm{N}_{\mathrm{d}}=\gamma_{\mathrm{G}} \mathrm{N}_{\mathrm{G}}+\gamma_{\mathrm{Q}} \mathrm{N}_{\mathrm{q}}
$$

où $\mathrm{N}_{G}$ est la sollicitation permanente (charges extérieures + poids propre de la semelle); $N_{Q^{\prime}}$ la sollicitation variable (dans cette étude, aucune sollicitation variable n'est prise en compte) ; $\gamma_{c}$, le facteur partiel de sécurité appliqué sur les sollicitations permanentes ; $\gamma_{0}$, le facteur partiel de sécurité appliqué sur les sollicitations variables. Les valeurs des facteurs partiels de sécurité sont données dans les tableaux VI et VII.

Pour les vérifications à l'état limite de service, tous les coefficients partiels de sécurité sont généralement égaux à un. Les déplacements de la fondation doivent être calculés et comparés aux valeurs limites admissibles permettant le bon fonctionnement de la structure. Les vérifications à l'état limite de service jouent un rôle important et décisif dans le dimensionnement final des ouvrages.

\section{Cas de l'Eurocode 7 (2004)}

D’après l'Eurocode 7 (2004), il convient de vérifier le dimensionnement des éléments structuraux (fondations superficielles) à l'état limite ultime (STR) soumis à des actions géotechniques, et la résistance du terrain (GEO) en utilisant l'une des trois approches suivantes complétées, pour les actions géotechniques et les résistances, par l'EN 1997 (tableau VI). Ces approches diffèrent par la façon dont elles distribuent les facteurs partiels de sécurité entre les actions, les effets des actions, les propriétés des matériaux et les résistances.

\begin{tabular}{|c|c|c|c|c|}
\hline \multicolumn{5}{|c|}{ Équilibres STR et GEO (fondations superficielles) } \\
\hline Approches & \multicolumn{2}{|c|}{ Approche 1} & Approche 2 & Approche 3 \\
\hline Combinaisons & $\mathrm{A} 1+\mathrm{M} 1+\mathrm{R} 1$ & $\mathrm{~A} 2+\mathrm{M} 2+\mathrm{R} 1$ & $\mathrm{~A} 1+\mathrm{M} 1+\mathrm{R} 2$ & $\mathrm{~A} 1 \mathrm{ou} \mathrm{A} 2+\mathrm{M} 2+\mathrm{R} 3$ \\
\hline \multicolumn{5}{|c|}{ Facteurs partiels pour les actions ou effets des actions (A) } \\
\hline $\begin{array}{l}\text { Action permanente } \\
\text { défavorable } \gamma_{G}\end{array}$ & 1,35 & 1,00 & 1,35 & 1,35 ou 1,00 \\
\hline $\begin{array}{l}\text { Action permanente } \\
\text { favorable } \gamma_{C}\end{array}$ & 1,00 & 1,00 & 1,00 & 1,00 \\
\hline $\begin{array}{l}\text { Action variable } \\
\text { défavorable } \gamma_{0}\end{array}$ & 1,50 & 1,30 & 1,50 & 1,50 ou 1,30 \\
\hline \multicolumn{5}{|c|}{ Facteurs partiels pour les paramètres de sol (M) } \\
\hline $\begin{array}{l}\text { Angle de frottement } \\
\text { interne } \gamma_{\varphi}^{\prime}\end{array}$ & 1,00 & 1,25 & 1,00 & 1,25 \\
\hline Cohésion $\gamma_{\mathrm{C}}{ }^{\prime}$ & 1,00 & 1,25 & 1,00 & 1,25 \\
\hline Poids volumique $\gamma_{\gamma}$ & 1,00 & 1,00 & 1,00 & 1,00 \\
\hline \multicolumn{5}{|c|}{ Facteurs partiels de ia résistance (R) } \\
\hline Portance $\gamma_{R}$ & 1,00 & 1,00 & 1,4 & 1,00 \\
\hline
\end{tabular}

TABLEAU vI Valeurs des facteurs partiels de sécurité (Eurocode 7, 2004).

Values of partial safety factors (Eurocode 7, 2004).

\begin{tabular}{|c|c|c|c|c|c|c|}
\hline & $\gamma_{\phi}$ & $\gamma_{c^{2}}$ & ${ }^{\prime}$ & NE. & 和 & 物 \\
\hline Approche $1 \mathrm{C}$ & 1,25 & 1,25 & 1,00 & 1,00 & 1,00 & 1,30 \\
\hline Approche 1B & 1,00 & 1,00 & 1,00 & 1,40 & 1,35 & 1,50 \\
\hline
\end{tabular}

TABLEAU WII Valeurs des facteurs partiels de sécurité d'après DIN 1054 (2005).

Values of partial safety factors from DIN 1054 (2005). 


\section{- Approche de calcul 1}

On doit vérifier qu'aucun état limite de rupture ou de déformation excessive ne sera atteint sous chacune des deux combinaisons de facteurs partiels suivantes :

- combinaison $1: \mathrm{A} 1+\mathrm{M} 1+\mathrm{R} 1$;

- combinaison 2 : A2 + M2 + R1.

Dans les combinaisons 1 et 2 , les facteurs partiels de sécurité sont appliqués aux actions et aux paramètres de résistance du terrain.

\section{- Approche de calcul 2}

On doit vérifier qu'aucun état limite de rupture ou de déformation excessive ne sera atteint avec la combinaison de facteurs partiels suivante:

- combinaison : A1 + M1 + R2.

Dans cette approche, les facteurs partiels de sécurité sont appliqués aux actions ou aux effets des actions et aux résistances du terrain.

\section{- Approche de calcul 3}

On doit vérifier qu'aucun état limite de rupture ou de déformation excessive ne sera atteint avec la combinaison de facteurs partiels suivante:

- combinaison : A1 ou A2 + M2 + R3.

Dans cette approche, les facteurs partiels de sécurité sont appliqués aux actions ou aux effets des actions et aux paramètres de résistance du terrain.

Dans l'approche 1, il y a deux combinaisons de calcul. Toutefois, dans cette étude, on a considéré seulement la combinaison 2 « $\mathrm{A} 2+\mathrm{M} 2+\mathrm{R} 1$ ) (tableau VI), car dans la combinaison 1 « A1 + M1 + R1 ) les facteurs partiels de sécurité sont appliqués uniquement sur les actions (A). Il s'agit pour ce problème de semelle des états limites de rupture ou de déformation excessive d'un élément de structure ou du terrain (STR et GEO), d'après la définition des états limites ultimes citée plus haut. De plus, cette combinaison donnerait des largeurs de semelles trop petites. Néanmoins, la combinaison 1 (A1 + M1 + R1) est également obligatoire pour le dimensionnement final.

Pour l'approche 3, on vérifie uniquement la combinaison " $\mathrm{A} 1+\mathrm{M} 2+\mathrm{R} 3$ ). La raison est que l'ensemble A2 s'applique uniquement aux actions géotechniques (et A1 aux actions de/sur la structure) ; il n'y a pas deux combinaisons possibles comme pour l'Approche de calcul 1 (tableau VI).

Les approches de l'Eurocode 7 (2004) sont désignées par l'abréviation EC 7 (approche 1 ou 2 ou 3).

\section{Cas des règles DIN 1054 (2005)}

DIN 1054 (2005) est la nouvelle norme allemande qui détermine les modalités de vérification de sécurité des ouvrages géotechniques en se référant à l'Eurocode 7 (2004) en tenant compte de la notion des facteurs partiels de sécurité. La norme DIN 1054 (2005) divise l'analyse de l'état limite ultime en trois approches.

- Approche $1 \mathrm{~A}$ : état limite ultime de perte de position. Défaillance d'une structure à travers une perte d'équilibre sans rupture (exemple : poussée d'Archimède).

- Approche $1 \mathbf{B}$ : état limite ultime de rupture d'une structure ou d'éléments constituant une structure ou du sol (exemple : capacité portante des fondations superficielles).
- Approche 1C : état limite ultime de rupture d'ensemble « structure et sol " (exemple : stabilité d'ensemble d'un mur de soutènement ancré).

Pour chaque cas, la norme recommande une combinaisun de calcul avec des coefficients partiels de sécurité. Dans le cas de l'étude de la stabilité des fondations superficielles vis-à-vis du poinçonnement, Ia norme DIN 1054 (2005) recommande de faire les vérifications par l'approche $1 \mathrm{~B}$, car elle est plus critique que l'approche 1C. L'approche $1 \mathrm{~A}$ n'est pas applicable dans ce problème. Dans l'approche $1 \mathrm{~B}$, les facteurs partiels de sécurité sont appliqués sur les forces, alors que dans l'approche $1 \mathrm{C}$ ils sont appliqués sur les paramètres du sol $\left(\gamma, \varphi^{\prime}, c^{\prime}\right)$ (tableau VII).

Les approches $1 \mathrm{~B}$ et $1 \mathrm{C}$ de la norme DIN 1054 (2005) correspondent respectivement aux approches 2 et 1 (combinaison 2) de l'Eurocode 7 (2004) (tableaux VI et VII). Pour cette raison, on étudie seulement les trois approches de l'Eurocude 7 (tableau VI)

- approche 1 (combinaison $2: \mathrm{A} 2+\mathrm{M} 2+\mathrm{B} 1)$;

- approche 2 (combinaison: $\mathrm{A} 1+\mathrm{M} 1+\mathrm{R} 2)$;

- approche 3 (combinaison: $\mathrm{A} 1+\mathrm{M} 2+\mathrm{R} 3$ ).

\section{Exemples numériques}

On considère toujours le même exemple.

\section{- Approche $1(\mathrm{~A} 2+\mathrm{M} 2+\mathrm{R} 1)$}

D'après le tableau VI $\varphi_{\mathrm{d}}=\frac{\arctan \varphi_{\mathrm{k}}^{\prime}}{\gamma_{\Phi^{\prime}}}=\frac{\arctan 30}{1,25}=24,79 \mathrm{de}-$ grés et $\mathrm{c}_{\mathrm{d}}^{\prime}=\frac{\mathrm{c}_{\mathrm{k}}^{\prime}}{\gamma_{\mathrm{c}^{\prime}}}=\frac{10}{1,25}=8 \mathrm{kPa}$, ce qui donne d'après le tableau Il les facteurs de portance suivants: $N_{4}=10,43$; $\mathrm{N}_{y}=8,71$ et $\mathrm{N}_{c}=20,42$. Ces valeurs sont reportées dans les équations (15) et (26). On obtient ainsi la capacité portante de calcul: $R_{d}=(87,1 \mathrm{~B}+476,26) \cdot \mathrm{B}$

D'après le tableau $\mathrm{VI}: \gamma_{\mathrm{G}}=1$.

La sollicitation de calcul d'après l'équation (27) vaut :

$$
N_{\mathrm{e} 1}=\gamma_{G} \cdot \mathrm{N}_{\mathrm{G}}=1 \cdot\left(\mathrm{Q}+\gamma_{\text {berr } n} \cdot \text { B.D. D. } 1\right)=290+36 \mathrm{~B} .
$$

En remplaçant les expressions de $R_{c i}$ et $N_{i d}$ dans l'équation (24), un obtient l'inéquation suivante:

$$
87,1 \mathrm{~B}^{\mathrm{e}}+440,62 \mathrm{~B}-290 \geq 0
$$

d'où l'on tire la largeur minimale de la semelle filante $\mathrm{B}=0,59 \mathrm{~m}$ (voir tableau VIII).

\section{- Approche $2(\mathrm{~A} 1+\mathrm{M} 1+\mathrm{R} 2)$}

$D^{\prime}$ après le tableau VI : $\gamma_{\Phi^{\prime}}=\gamma_{C^{\prime}}=1$ et $\gamma_{R}=1,4$.

D'après le tableau II : pour $\varphi_{k}=30$ degrés, on a $\mathrm{N}_{\mathrm{q}}=18,4 ; \mathrm{N}_{\mathrm{y}}=20,\left(19\right.$ et $\mathrm{N}_{\mathrm{c}}=30,14$.

La capacité portante de calcul d'après les équations (15) et (25) est égale à

$$
\mathrm{R}_{\mathrm{s} 1}=(200,9 \mathrm{~B}+853,4) \cdot \mathrm{B} / 1,4 \text {. }
$$

D'après le tableau VI : $\gamma_{\mathrm{C}}=1.35$.

La sollicitation de calcul d'après l'équation (27) vaut

$$
\mathrm{N}_{\mathrm{j}}=\gamma_{\mathrm{G}}\left(\mathrm{Q}+\gamma_{\text {béton }} \text { B.D.1) }=1,35 \cdot(290+36 \mathrm{~B}) .\right.
$$

En remplaçant les expressions de $R_{d}$ et $N_{d}$ dans l'équation (24), on obtient l'inéquation suivante : 2()(),9 B² $+785,36 \mathrm{~B}-548,1 \geq 0$

d'où l'on tire la largeur minimale de la semelle filante $B$ $=0,61 \mathrm{~m}$ (voir tableau VIII). 
- Approche $3(\mathrm{~A} 1+\mathrm{M} 2+\mathrm{R} 3)$

D'après le tableau VI: $\varphi_{\mathrm{d}}^{\prime}=\frac{\arctan \varphi_{\mathrm{k}}^{\prime}}{\gamma_{\varphi^{\prime}}}=\frac{\arctan 30}{1,25}=24,79$ degrés et $c_{\mathrm{d}}^{\prime}=\frac{\mathrm{c}_{\mathrm{k}}^{\prime}}{\gamma_{\mathrm{C}^{\prime}}}=\frac{10}{1,25}=8 \mathrm{k} \cdot \mathrm{Pa}$, ce qui conduit aux facteurs de portance suivants d'après le tableau II : $\mathrm{N}_{\mathrm{q}}=10,43$; $N_{y}=8,71$ et $N_{c}=20,42$. Ces valeurs sont ensuite reportées dans les équations (15) et (26). On obtient ainsi la capacité portante de calcul :

$\mathrm{R}_{\mathrm{d}}=(87,1 \mathrm{~B}+476,26) \cdot \mathrm{B}$.

D'après le tableau VI : $\gamma_{G}=1,35$ et la sollicitation de calcul est estimée par la relation (27):

$$
\mathrm{N}_{\mathrm{d}}=\gamma_{\mathrm{G}} \cdot\left(\mathrm{Q}+\gamma_{\text {béton: }} \text {. B.D.1) }=1,35 \cdot(290+36 \mathrm{~B})\right. \text {. }
$$

En remplaçant les expressions de $R_{d}$ et $N_{d}$ dans l'équation (24) on aboutit à l'équation :

$$
87,1 \mathrm{~B}^{2}+427,66 \mathrm{~B}-391,5 \geq 0
$$

d'où l'on déduit la largeur minimale de la semelle filante $\mathrm{B}=0,79 \mathrm{~m}$ (voir tableau VIII).

\section{Interprétation des résultats}

Les résultats des calculs des largeurs minimales des semelles étudiées pour différents cas de charges, différents types de sol et d'après différentes approches sont regroupés dans les tableaux VIII à XIII. L'interprétation de ces résultats peut être résumé comme suit:

TABLEAU vil Largeur minimale $B|\mathrm{~m}|$ d'une semelle filante d'après différentes approches.

Charge verticale centrée $Q=290 \mathrm{kN} / \mathrm{ml}$.

\begin{tabular}{|c|c|c|c|c|c|}
\hline \multirow[b]{3}{*}{$\varphi^{\prime}[$ degrés $] / c^{\prime}[\mathrm{kPa}]$} & \multicolumn{2}{|c|}{ Approches déterministes } & \multirow{2}{*}{\multicolumn{3}{|c|}{$\begin{array}{l}\text { Approches serni-probahilistes } \\
\text { Approches ite l'Eurocorie } 7(2004)\end{array}$}} \\
\hline & \multirow{2}{*}{ 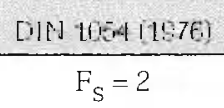 } & \multirow{2}{*}{$\frac{\text { DTU } 13.12(1988)}{F_{S}-2}$} & & & \\
\hline & & & Approche 1 & Approche 2 & Approche 3 \\
\hline $40 / 0$ & 0,27 & 0,27 & 0,31 & 0,26 & 0,40 \\
\hline $35 / 0$ & 0,50 & 0,50 & 0,52 & 0,48 & 0,68 \\
\hline $35 / 5$ & 0,43 & 0,43 & 0,44 & 0,41 & 0,58 \\
\hline $30 / 10$ & 0,64 & 0,63 & 0,59 & 0,61 & 0,79 \\
\hline $25 / 20$ & 0,79 & 0,77 & 0,66 & 0,75 & 0,90 \\
\hline $20 / 35$ & 0,86 & 0,83 & 0.66 & 0.81 & 0.91 \\
\hline
\end{tabular}

Minimal width B [m] of strip footing from different approaches. Vertical centered load $Q=290 \mathrm{kN} / \mathrm{ml}$

TABLEAU IX Largeur minimale $B|\mathrm{~m}| \mathrm{d}^{\prime}$ une semelle filante d'après différentes approches.

Charge centrée inclinée $Q=400 \mathrm{kN} / \mathrm{ml}$ et inclinaison $\delta=10$ degrés.

Minimal width B [m] of strip footing from different approaches.

\begin{tabular}{|c|c|c|c|c|c|}
\hline \multirow{3}{*}{$\underline{\varphi^{\prime}[\text { degrés }] / \mathrm{c}^{\prime}[\mathrm{kPa}]}$} & \multicolumn{2}{|c|}{ Approches déterministes } & \multirow{2}{*}{\multicolumn{3}{|c|}{$\begin{array}{l}\text { Approches semi-probabilistes } \\
\text { Approches de l'Eurocode } 7 \text { (2004) }\end{array}$}} \\
\hline & \multirow{2}{*}{$\frac{\text { DIN } 1054(1976)}{F_{S}=2}$} & \multirow{2}{*}{$\frac{\text { DTU } 13.12(1988)}{F_{S}=2}$} & & & \\
\hline & & & Approche 1 & Approche 2 & Approche 3 \\
\hline $40 / 0$ & 0,52 & 0,46 & 0,59 & 0,50 & 0,77 \\
\hline $35 / 0$ & 0,95 & 0,86 & 0,99 & 0,91 & 1,28 \\
\hline $35 / 5$ & 0,83 & 0,73 & 0,86 & 0,79 & 1,13 \\
\hline $30 / 10$ & 1,25 & 1,10 & 1,19 & 1,19 & 1,57 \\
\hline $25 / 20$ & 1,63 & 1,37 & 1,41 & 1,54 & 1,91 \\
\hline $20 / 35$ & 1,92 & 1,48 & 1,53 & 1,80 & 2,11 \\
\hline
\end{tabular}

Inclined centered load $Q=400 \mathrm{kN} / \mathrm{ml}$ and inclination $\delta=10$ degrees.

TABLEAU $x$ Largeur minimale $B|m|$ d'une semelle fillante d'après différentes approches.

Charge verticale excentrée $Q=800 \mathrm{kN} / \mathrm{ml}$ et excentricité e $=B / 6$.

Minimal width B [m] of strip footing from different approaches.

Eccentric vertical load $\mathrm{Q}=800 \mathrm{kN} / \mathrm{ml}$ and eccentricity $\mathrm{e}=\mathrm{B} / 6$.

Approches déterministes IHN 1054 (1976)
DTU 13.12 (1988)

\begin{tabular}{cccccc}
$\varphi^{\prime}[$ degrés]/c'[kPa] & $\mathrm{F}_{\mathrm{S}}=2$ & $\mathrm{~F}_{\mathrm{S}}=2$ & Approche 1 & Approche 2 & Approche 3 \\
$40 / 0$ & 0,95 & 0,96 & 1,09 & 0,91 & 1,39 \\
$35 / 0$ & 1,68 & 1,68 & 1,76 & 1,60 & 2,24 \\
$35 / 5$ & 1,49 & 1,50 & 1,56 & 1,43 & 2,00 \\
$30 / 10$ & 2,22 & 2,21 & 2,13 & 2,12 & 2,76 \\
$25 / 20$ & 2,88 & 2,83 & 2,50 & 2,73 & 3,30 \\
$20 / 35$ & 3,31 & 3,21 & 2,60 & 3,13 & 3,51 \\
\hline
\end{tabular}

Approches semi-probabilistes Approches de l'Eurocode 7 (2004) 
TABLEAU X] Largeur minimale B [m] d'une semelle carrée d'après différentes approches. Charge verticale centrée $Q=290 \mathrm{kN}$.

Minimal width B [m] of square footing from different approaches. Centered vertical load $\mathrm{Q}=290 \mathrm{kN}$

\begin{tabular}{|c|c|c|c|c|c|}
\hline & \multicolumn{2}{|c|}{ Approches déterministes } & \multirow{2}{*}{\multicolumn{3}{|c|}{$\begin{array}{l}\text { Approches semi-probabilistes } \\
\text { Approches de l'Eurocode } 7 \text { (2004) }\end{array}$}} \\
\hline & DIN 1054 (1976) & DTU $13.12(1988)$ & & & \\
\hline$\varphi^{\prime}[$ degrés]/c'[kPa] & $F_{S}=2$ & $F_{S}=2$ & Approche 1 & Approche 2 & Approche 3 \\
\hline $40 / 0$ & 0,41 & 0,51 & 0,45 & 0,40 & 0,52 \\
\hline $35 / 5$ & 0,53 & 0,63 & 0,55 & 0,52 & 0.64 \\
\hline $30 / 10$ & 0,67 & 0.77 & 0,65 & 0,65 & 0,76 \\
\hline $25 / 20$ & 0,75 & 0,84 & 0,69 & 0,73 & 0.81 \\
\hline $20 / 35$ & 0,79 & 0,85 & 0,70 & 0,76 & 0,82 \\
\hline
\end{tabular}

TABLEAU XII Largeur minimale B [m] d'une semelle carrée d'après différentes approches.

Charge centrée inclinée $Q=400 \mathrm{kN}$ et inclinaison $\delta=10$ degrés.

Minimal width B [m] of square footing from different approaches.

Inclined centered load $\mathrm{Q}=400 \mathrm{kN}$ and inclination $\delta=10$ degrees.

\begin{tabular}{|c|c|c|c|c|c|}
\hline & \multicolumn{2}{|c|}{ Approches déterministes } & \multirow{2}{*}{\multicolumn{3}{|c|}{$\begin{array}{l}\text { Approches semi-probabilisĩes } \\
\text { Approches de l'Eurocode } 7 \text { (ख004) }\end{array}$}} \\
\hline & DIN $1054(1976)$ & DTU 13.12 (1988) & & & \\
\hline$\varphi^{\prime}[$ degrés $] / \mathrm{c}^{\prime}[\mathrm{kPa}]$ & $F_{S}=2$ & $F_{S}=2$ & Approche 1 & Approche 2 & Approche 3 \\
\hline $40 / 0$ & 0,59 & 0,67 & 0,64 & 0,57 & 0,75 \\
\hline $35 / 5$ & 0,77 & 0,85 & 0,79 & 0,74 & 0,92 \\
\hline $30 / 10$ & 0,96 & 1,03 & 0,94 & 0,94 & 1,11 \\
\hline $25 / 20$ & 1,10 & 1,12 & 1,03 & 1,07 & 1,21 \\
\hline $20 / 35$ & 1,19 & 1,14 & 1,07 & 1,15 & 1,27 \\
\hline
\end{tabular}

TABLEAU XIII Largeur minimale B [m] d'une semelle carrée d'après différentes approches.

Charge verticale excentrée $Q=800 \mathrm{kN}$ et excentricité $\mathrm{e}=\mathrm{B} / 6$.

Minimal width B [m] of square footing from different approaches. Eccentric vertical luad $Q=800 \mathrm{kN}$ and eccentricity e $=B / 6$.

\begin{tabular}{|c|c|c|c|c|c|}
\hline \multirow{3}{*}{$\frac{\varphi^{\prime}[\text { degrés }] / c^{\prime}[\mathrm{kPa}]}{}$} & \multicolumn{2}{|c|}{ Approches dérerministes } & \multirow{2}{*}{\multicolumn{3}{|c|}{$\begin{array}{l}\text { Approches semj-probabilistes } \\
\text { Approches de l'Eurocode } 7 \text { (2004) }\end{array}$}} \\
\hline & \multirow{2}{*}{$\frac{\text { DIN 1054 (1976) }}{F_{S}=2}$} & \multirow{2}{*}{$\frac{\text { DTU } 13.12(1 \mathrm{BB} 8)}{\mathrm{F}_{\mathrm{S}}=2}$} & & & \\
\hline & & & Approche 1 & Approche 2 & Approche 3 \\
\hline $40 / 0$ & 0,87 & 0,99 & 0,94 & 0,85 & 1.09 \\
\hline $35 / 5$ & 1,12 & 1,26 & 1,15 & 1,09 & 1,34 \\
\hline $30 / 10$ & 1,40 & 1,54 & 1,37 & 1,36 & 1,59 \\
\hline $25 / 20$ & 1,58 & 1,70 & 1,47 & 1,54 & 1.72 \\
\hline $20 / 35$ & 1,67 & 1,76 & 1,48 & 1,62 & 1,74 \\
\hline
\end{tabular}

- l'approche 3 (EC 7) conduit aux plus grandes largeurs de semelles, à cause de l'application simultanée des facteurs partiels de sécurité sur les forces et sur les paramètres du sol (tableau VI) ;

- les approches DIN $1054\left(F_{s}=2\right)$ et DTU $13.12\left(F_{s}=2\right)$ fournissent généralement des largeurs de semelles peu différentes de celles calculées d'après les approches de l'EC 7 (Approches 1 et 2 ) à l'exception du cas des sols à forte cohésion $(\mathrm{c} \geq 20 \mathrm{kPa})$;

- l'approche 2 (EC 7) donne les plus petites largeurs des semelles pour les sols pulvérulents et de faible cohésion ( $\mathrm{C}^{\prime} \leq 10 \mathrm{kPa}$ ), alors que l'approche 1 (EC 7) donne les plus petites largeurs de semelles pour les sols à moyenne et forte cohésion ( $\left.\mathrm{c}^{\prime}>10 \mathrm{kPa}\right)$.

\section{6}

\section{Calcul de l'indice de fiabilité}

Le calcul de l'indice de fiabilité a été réalisé à l'aide du logiciel ZUVAN développé à l’Université de Weimar (Allemagne) et fondé sur la théorie de fiabilité de premier ordre (Belabed, 1996) en utilisant soit l'algorithme de Späthe (Späthe, 1987), soit l'algorithme de Rackwitz (Rackwitz, 1978; Fießler et al., 1976). Le principe de cette méthode est expliqué plus haut. On doit d'abord implanter dans ce logiciel l'équation d'état limite pour chaque approche. A chaque valeur de largeur B calculée auparavant (tableaux VIII à XIII), on calcule l'indice 
de fiabilité $\beta$ correspondant. Le tableau $V$ regroupe les variables aléatoires et leurs propriétés statistiques nécessaires pour ces calculs.

- Exemple d'une semelle carrée avec une charge centrée inclinée $(\mathrm{Q}=400 \mathrm{kN}, \delta=$ 10 degrés) - Approche 2 de l'Eurocode 7

Pour cet exemple, l'équation (24) est implantée dans le programme ZUVAN en tenant compte des équations (17), (25) et (27). Les données statistiques des paramètres (tableau V) ainsi que la largeur de la semelle calculée auparavant sont introduites dans le programme.

Pour $\varphi^{\prime}=40$ degrés et $c^{\prime}=0$, le tableau XII indique une largeur de semelle égale à $0,57 \mathrm{~m}$. Le calcul itératif conduit alors à un indice de fiabilité $\beta=2,3$ (Fig. 11). Les détails des résultats du calcul probabiliste, au moyen du logiciel ZUVAN, sont regroupés dans le tableau XIV.

Pour $\varphi^{\prime}=35$ degrés et $\mathrm{c}^{\prime}=5 \mathrm{kPa}$, le tableau XII fournit une largeur de semelle égale à $0,74 \mathrm{~m}$; d'où, un indice de fiabilité $\beta=2,671$ (Fig. 11).

On procède de la même manière pour les autres approches.

Les résultats des calculs des indices de fiabilité pour tous les cas ont été regroupés dans les figures 7 à 11 pour différents types de sols d'après différentes appro

tableAU XIV Résultats d'un exemple de calcul de l'indice de fiabilité.

Results of the calculation of the reliability index.

\begin{tabular}{cccc} 
Paramètre & $\begin{array}{c}\text { Valeur } \\
\text { caractéristique }\end{array}$ & $\begin{array}{c}\text { Valeur } \\
\text { de calcul }\end{array}$ & $\begin{array}{c}\text { Facteur } \\
\text { de sensibilité }\end{array}$ \\
\hline$\varphi^{\prime}$ [degrés] & 40 & 31,11 & 0,9659 \\
$\mathrm{C}^{\prime}[\mathrm{kPa}]$ & 0 & 0 & 0 \\
$\gamma\left[\mathrm{kN} / \mathrm{m}^{3}\right]$ & 20 & 19,74 & 0,1116 \\
$\mathrm{Q}[\mathrm{kN}]$ & 400 & 419,40 & $-0,2109$ \\
$\delta[$ degrés] & 10 & 10,23 & $-0,1003$ \\
$\beta[-]$ & \multicolumn{4}{|c}{ 2,30 (voir Fig. 11) } \\
\hline
\end{tabular}

Semelle filante. Charge verticale centrée $Q=290 \mathrm{kN} / \mathrm{m}$

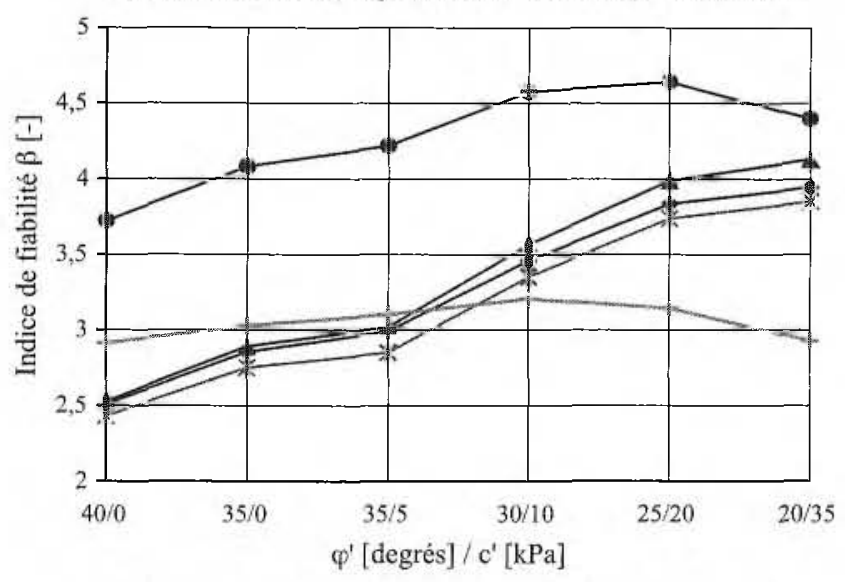

\footnotetext{
$\longrightarrow$ DTU $13.12\left(\mathrm{~F}_{\mathrm{s}}=2\right)$

*- EC7 (Approche 2)

- EC 7 (Approche 1)
}

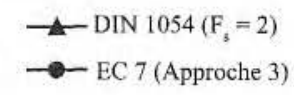

FiG. 7 Indice de fiabilité $\beta$ d'après différentes approches. Charge verticale centrée $\mathrm{Q}=290 \mathrm{kN} / \mathrm{ml}$.

Reliability index $\beta$ from different approaches. Centered vertical load $Q=290 \mathrm{kN} / \mathrm{ml}$. ches. L'interprétation de ces résultats a permis de tirer les constatations suivantes

- l'approche 3 (EC 7) sous-estime la sécurité en donnant les plus grandes largeurs de semelles et les plus grands indices de fiabilité. Le niveau de sécurité correspondant est inhomogène sur toute la fourchette des sols étudiés (Fig. 7). Par suite, les résultats des indices de fiabilité pour cette approche ne sont pas présentés pour les autres cas (Fig. 8 à 11);

- le niveau de sécurité obtenu pour l'approche 1 (EC 7), dont les facteurs partiels de sécurité sont appliqués sur les paramètres du sol, est relativement homogène le long de la fourchette des sols étudiés en comparaison avec les approches dont les facteurs de sécurité sont appliqués sur les forces (approches 2 (EC 7), DIN 1054 $\left(F_{S}=2\right)$ et DTU $\left.13.12\left(F_{S}=2\right)\right)$. Ces dernières approches

Semelle filante. Charge centrée inclinée $Q=400 \mathrm{kN} / \mathrm{m}$

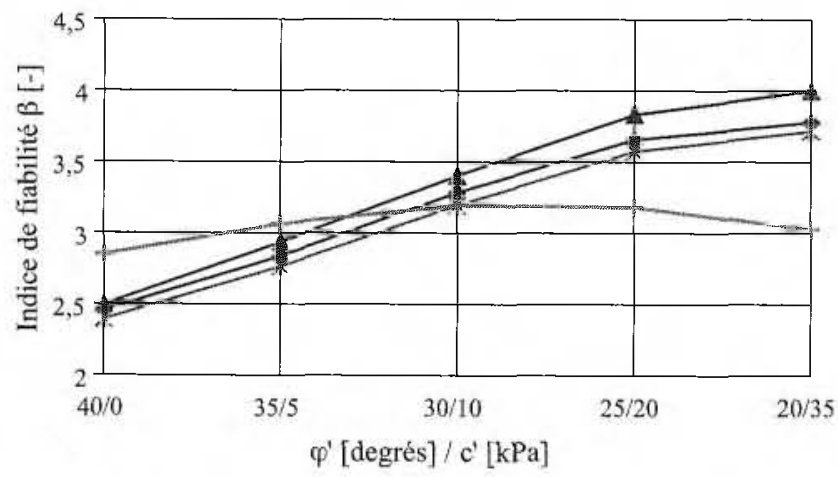

$$
\begin{aligned}
& \longrightarrow \text { DTU } 13.12\left(\mathrm{~F}_{\mathrm{s}}=2\right) \\
& \text { * EC 7 (Approche 2) }
\end{aligned}
$$

FIG. 8 Indice de fiabilité $\beta$ d'après différentes approches. Charge centrée inclinée $\mathrm{Q}=400 \mathrm{kN} / \mathrm{ml}$ et inclinaison $\delta=10$ degrés. Reliability index $\beta$ from different approaches. Inclined centered load $Q=400 \mathrm{kN} / \mathrm{ml}$ and inclination $\delta=10$ degrees.

\section{Semelle filante. Charge verticale excentrée $Q=800 \mathrm{kN} / \mathrm{m}$}

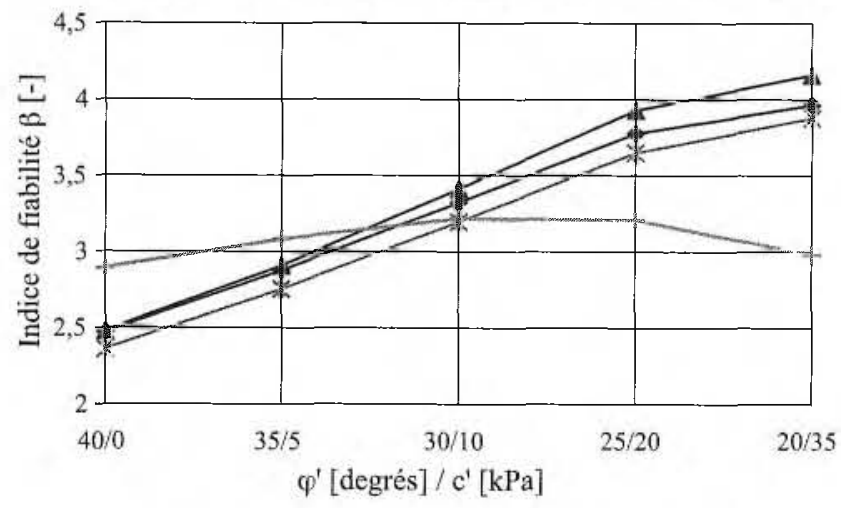

$$
\begin{aligned}
& \longrightarrow \text { DTU } 13.12\left(\mathrm{~F}_{\mathrm{s}}=2\right) \\
& \text { - } \mathrm{EC} 7 \text { (Approche 2) DIN } 1054\left(\mathrm{~F}_{\mathrm{s}}=2\right) \\
& \text { - EC 7 (Approche 1) }
\end{aligned}
$$

FIG. 9 Indice de fiabilité $\beta$ d'après différentes approches. Charge verticale excentrée $\mathrm{Q}=800 \mathrm{kN} / \mathrm{ml}$ et excentricité $\mathrm{e}=\mathrm{B} / 6$. Reliability index $\beta$ from different approaches. Eccentric vertical load $Q=800 \mathrm{kN} / \mathrm{ml}$ and eccentricity e $=\mathrm{B} / 6$. 
Semelle carrée. Charge verticale centrée $Q=290 \mathrm{kN}$

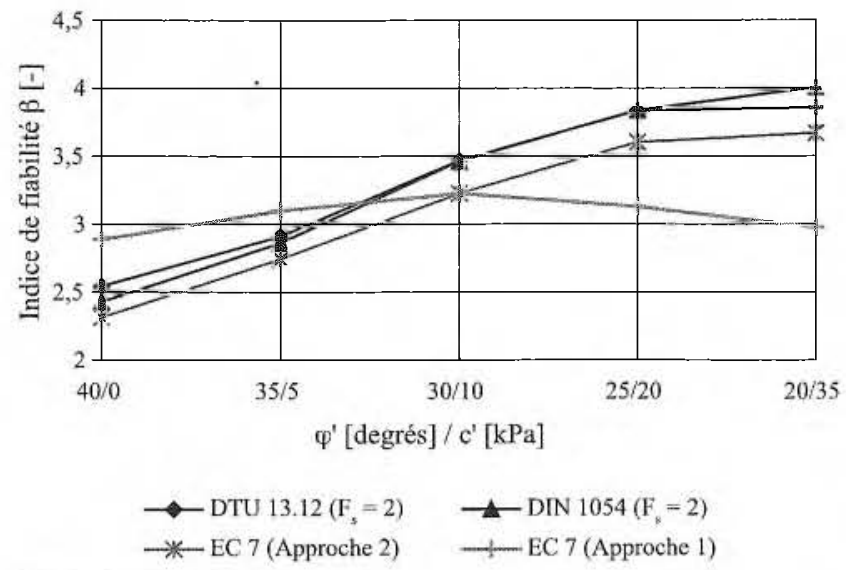

F16. 10. Indice de fiabilité $\beta$ d'après différentes approches. Charge verticale centrée $\mathrm{Q}=290 \mathrm{kN}$.

Reliability index $\beta$ from different approaches. Vertical centered load $Q=290 \mathrm{kN}$.

présentent la même tendance pour les valeurs de $\beta$ (Figs. 7 à 11). Celles obtenues avec l'approche 1 sont proches des valeurs cibles $\beta=3$ (Figs. 7 à 11) ;

- dans tous les cas étudiés, les approches de l'Eurocode 7 (approches 1 et 2) sont de loin les plus défavorables. Elles donnent le minimum de sécurité (Figs. 7 à 11) et les plus petites dimensions des semelles (Tableaux VIII à XIII). L'approche 2 est la plus défavorable pour les sols pulvérulents et de faible cohésion $\left(\mathrm{C}^{\prime} \leq 10 \mathrm{kPa}\right)$. L'approche 1 (combinaison 2) est la plus défavorable pour les sols à moyenne et forte cohésion $\left(c^{\prime}>10 \mathrm{kPa}\right)$.

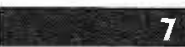

\section{Conclusion}

La vérification à l'état ultime de la stabilité des fondations superficielles vis-à-vis du poinçonnement se fait traditionnellement en appliquant un coeffi-
Semelle carrée. Charge centrée inclinée $Q=400 \mathrm{kN}$

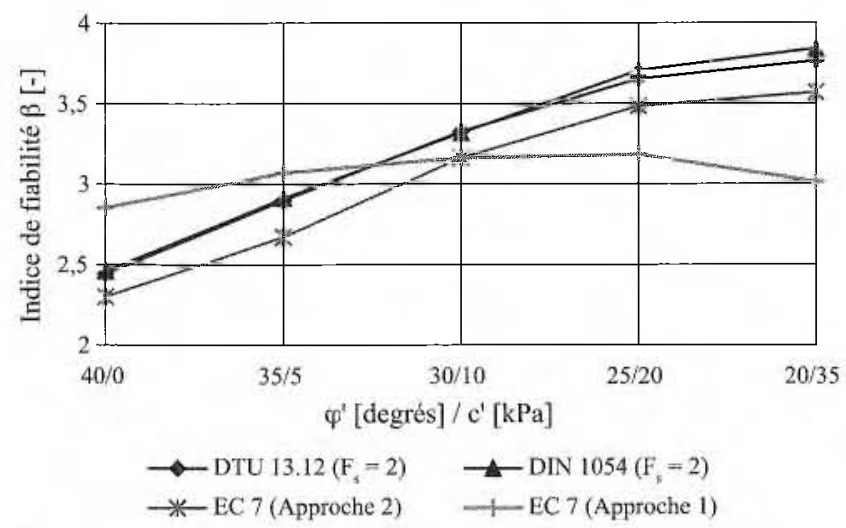

FIG. 11. Indice de fiabilité $\beta$ d'après différentes approches. Charge centrée inclinée $\mathrm{Q}=400 \mathrm{kN}$

Reliability index $\beta$ from different approaches. Inclined centered load: $\mathrm{Q}=400 \mathrm{kN}$.

cient global de sécurité $F_{S}=2$ sur la charge limite calculée par la théorie de Prandtl. Dans cette étude, on a présenté une nouvelle analyse du problème fondée sur la théorie probabiliste (EC 7) en appliquant des facteurs partiels de sécurité sur les forces (approche 2) ou sur les paramètres de sol (approche 1). Une analyse comparative avec les approches déterministes nationales a été effectuée. Les approches 1 et 2 de l'Eurocode 7 sont les plus défavorables. Le calcul de l'indice de fiabilité est très important pour l'évaluation de la sécurité. L'approche semi-probabiliste est un moyen d'uniformiser les codes et les normes traitant le calcul des structures en remplaçant les facteurs globaux empiriques de sécurité par des facteurs partiels de sécurité tenant compte de la dispersion aléatoire des paramètres. De plus, cette approche assure un emploi souvent économique des matériaux. Les vérifications à l'état limite de service sont également importantes dans le dimensionnement final des ouvrages. 
Abramson L.W., Lee T.S., Sharma S., Boyce G.M. - Slope stability and stabilization methods, 2002 Second edition, J. Wiley \& Sons, Inc.

Baguelin F., Kovarik J.B. - Une méthode de détermination des valeurs caractéristiques des paramètres géotechniques. Revue française de géotechnique, $n^{\circ} 93$, 2000 , p. 35-42.

Belabed L. - Standsicherheitsuntersuchung zweifach verankerter Stützwände mit der kinematischen Starrkörpermethode. Geotechnik n¹9, 1995, p. 171-174.

Belabed L. - Zuverlässigkeitsuntersuchung des Tragsystems \& mehrfach verankerte Stützwände ” mit probabilistischen Methoden. Thèse de doctorat, université de Weimar, Allemagne, 1996.

Belabed L. - Application du concept probabiliste de sécurité sur la vérification de la stabilité d'ensemble des murs de soutènement ancrés avec la cinématique des solides rigides. Revue française de géotechnique n 89, 1999a, p. 49-54

Belabed L. - Overall stability of anchored retaining walls with the probabilistic method. Proceeding of the international symposium on slope stability engineering, IS-Shikoku'99, Matsuyama/ Shikoku, Japan/ 8-11 Nov., 1999b, A.A.Balkema, Rotterdam/Brookfield, p. 1115-1120.

Bencheikh M. - Analyse probabiliste de la stabilité des fondations superficielles. Mémoire de Magister, Département de Génie civil, Université de Guelma, Algérie, 2005

Breitschaft G., Hanisch J. - Neues Sicherheitskonzept im Bauwesen aufgrund wahrscheinlichkeitstheoretischer Überlegungen. Folgerungen für den Grund- bau unter Einbeziehung der Probenahme und der Versuchsauswertung Vorträge der Baugrundtagung, Berlin, Herausgegeben von DGEG Essen, 1978 , p. 659-694.

Costet J., Sanglerat G. - Cours pratique de mécanique des sols, Tome 2. Dunod, Paris, 1983.

DIN 1054 - Sicherheitsnachweise im Erdund Grundbau. Beuth Verlag GmbH, Berlin, 1976.

DIN 1054 - Sicherheitsnachweise im Erdund Grundbau. Beuth Verlag GmbH, Berlin, 2005.

Ditlevsen O. - Model uncertainty in structural reliability. Structural safety 1, 1982 p. 73-86.

DTU 13.12 - Règles pour le calcul des fondations superficielles. Cahiers CSTB, AFNOR, Paris, 1988.

Eurocode 7 - Calcul géotechnique, partie 1 « Règles générales », 2004

Favre J.L. - Géotechnique, sécurité des ouvrages, risques. Technosup, Ellipses, 2004.

Fießler B., Hawranek H., Rackwitz R. Numerische Methoden für probabilistische Bemessungsverfahren und Sicherheitsnachweise. Heft 14, TU München, Allemagne, 1976.

Frank R. - Calcul des fondations superficiclles et profondes. Presses des Ponts et Chaussées, 2003

Gäßler G. - Vernagelte GeländesprüngeTragverhalten und Standsicherheit. Thèse de doctorat, Heft 108, université de Karlsruhe, Allemagne, 1987.

Genske D.D., Walz B. - Anwendung der probabilistischen Sicherheitstheorie au Grundbruchberechnungen nach DIN 4017. Geotechnik n 10, 1987, p. 53-66.
JCSS - Probabilistic model code. The Joint Committee on Structural Safety, 2001.

Magnan J.P., Baghery S. - Statistiques et probabilités en mécanique des sols, état des connaissances. Laboratoire central des ponts et chaussées, 1982

Nottrodt H.P. - Beitrag zur Einführung semiprobabilistischer Methoden in der Geotechnik. Thèse de doctorat, université de Weimar, Allemagne, 1990.

Orr T.L.L. - Selection of characteristic values and partial factors in geotechnical designs to Eurocode 7. Computers and Geotechnics 26, 2000, p. 263-279.

Pottharst R. - Erläuterungen des statistischen Sicherheitskonzepts am Beispiel des Grundbruchs. Vorträge der Baugrundtagung in Braunschweig, DGEG Essen, 1982, p. 9-47.

Rackwitz R. - Close Bounds for the reliabi lity of structural systems. SFB 96, Heft 29, TU München, Allemagne, 1978.

Soos P. - Zur Ermittlung der Bodenkennwerte mit Berücksichtigung von Streuung und Korrelation. Vorträge der Baugrundtagung in Braunschweig, DGEG Essen, 1982, p. 83-103.

Sørensen J.D. - Calibration of partial safety factors in Danish structural codes. JCSS workshop on reliability based code calibration, Zurich, Suisse, 21-22 mars 2002.

Späthe G. - Die Sicherheit tragender Baukonstruktionen. Verlag für Bauwesen Berlin, 1987.

Vrouwenvelder T. - Reliability based code calibration, the use of the JCSS Probabilistic model code. JCSS workshop on reliability based code calibration, Zurich, Suisse, 21-22 mars 2002. 